\title{
Star clusters as building blocks for dSph galaxy formation
}

\author{
P. Assmann, ${ }^{1}$ M. Fellhauer ${ }^{1}$ and M. I. Wilkinson ${ }^{2}$ \\ ${ }^{1}$ Departamento de Astronomía, Universidad de Concepción, Casilla 160-C, Concepción, Chile \\ email: [passmann, mfellhauer] @astro-udec.cl \\ ${ }^{2}$ Department of Physics \& Astronomy, University of Leicester, Leicester, UK \\ email: miw6@astro.le.ac.uk
}

\begin{abstract}
We study numerically the formation of dSph galaxies. Intense starbursts, e.g., in gas-rich environments, typically produce a few to a few hundred young star clusters within a region of just a few hundred pc. The dynamical evolution of these star clusters may explain the formation of the luminous component of dwarf spheroidal (dSph) galaxies. Here, we perform a numerical experiment to show that the evolution of star cluster complexes in dark-matter haloes can explain the formation of the luminous components of dSph galaxies.
\end{abstract}

Keywords. methods: $N$-body simulations, galaxies: dwarf, galaxies: halos

\section{Introduction}

Understanding the formation and evolution of galaxies is a fundamental goal in astronomy. Galaxies are believed (in the standard cosmological scenario) to build up by the merging of smaller constituents, namely dwarf galaxies. This scenario makes dwarf galaxies the basic building blocks of the structure formation in the Universe (e.g., Springel et al. 2005). Therefore, to understand galaxy formation and evolution on all scales, it is crucial to understand the formation and evolution of the smallest constituents first. Dwarf galaxies come in different shapes and dynamical states, e.g., dwarf disc galaxies, dwarf elliptical galaxies (with or without a nucleus), dwarf irregulars, ultracompact dwarfs and dwarf spheroidals (dSphs), to name a few. According to the standard scenario, all galaxies reside in an extended dark-matter (DM) halo, whereby dwarf galaxies should reside in the highest- $M / L$ (mass-to-light ratio) DM haloes (relative to their luminous, baryonic mass) of all galaxies.

We investigate numerically the initial conditions for the formation of dSph galaxies. Our model is based on the assumption that stars form in starbursts (Lada \& Lada 2003), producing star clusters within a region of just a few hundred pc. The dynamical evolution of these star clusters, i.e., their dissolution due to gas expulsion and subsequent merging in the centre of the DM halo, may explain the formation of dSph galaxies. Our numerical simulations are carried out using the particle mesh code SupERBox (Fellhauer et al. 2000), which allows us to keep track of many objects in one simulation. The distribution of the star clusters inside the central area of the DM halo follows a Plummer distribution, while we take two distinct types of DM haloes into account: a cusped Navarro, Frenk \& White (NFW; Navarro et al. 1997) and a cored Plummer profile (Plummer 1911). The setup of our simulation is as follows: the single star clusters are represented by Plummer spheres with a Plummer radius of $4 \mathrm{pc}$ and a cutoff radius of 25 pc. Each cluster has an initial mass of $10^{5} \mathrm{M}_{\odot}$ and an initial crossing time of $2.4 \mathrm{Myr}$, and is represented by 100000 particles. Fifteen of these clusters are placed in a star cluster (SC) complex that is modelled again as a Plummer distribution (i.e., positions and velocities according to the 
Plummer distribution function), now with the star clusters as particles. This Plummer distribution is given a Plummer radius of $25 \mathrm{pc}$ and a cutoff radius of $150 \mathrm{pc}$. The DM haloes have a scale length of $500 \mathrm{pc}$ and the mass enclosed within one scale length is $1.01 \times 10^{8} \mathrm{M}_{\odot}$, using 1000000 particles. For the DM-halo NFW profile (Plummer profile), we used a cutoff radius of $16 \mathrm{kpc}(2.5 \mathrm{kpc})$.

\section{Experimental results}

In Figure 1 top (bottom), we show a time sequence of surface-density contours of the star clusters travelling through the central area of the cored (cusped) DM halo, while expanding due to gas expulsion, collisions, etc. This leads to subsequent dissolution of the SCs and the distribution of their stars in the central area of the DM halo, building up the luminous component of the dSph galaxy.
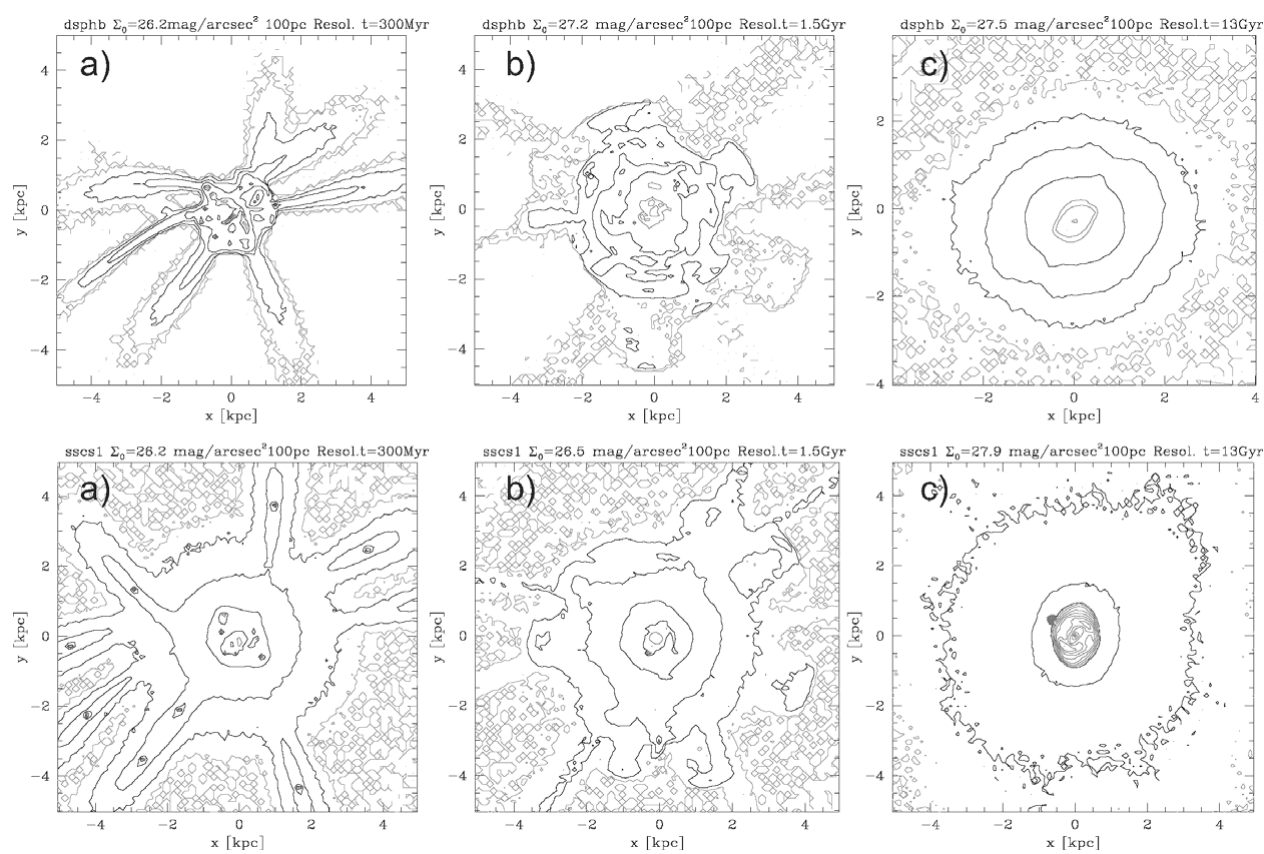

Figure 1. (top) dSph fomation based on star cluster dissolution in a cored DM halo. The surface density is shown at (a) $300 \mathrm{Myr}$, (b) $1.5 \mathrm{Gyr}$ and (c) 13 Gyr. (bottom) dSph fomation based on star cluster dissolution in a cusped DM halo. The surface density is shown at the same time steps as in the top panel.

In Figure 2 for both scenarios of DM haloes, the outer contours of our objects are rather smooth, as has been observed in most of the larger dwarf galaxies close to the Milky Way. However, it is interesting to note that there are still some fluctuations visible in the central parts, and observations of these fluctuations might corroborate our model for $\mathrm{dSph}$ galaxy formation. We note that in the cusped simulation, the stars are more centrally concentrated. In the middle panels, we can see that the velocity dispersion is still about 50\% higher than expected considering the observations of Draco or the Ursa Minor dSph, but focusing on the core of our simulated objects, in the cusped simulation the dispersion velocities are lower than the cored simulation. The mean velocities per pixel, shown in the right panels, show areas which differ by $\sim 9 \mathrm{~km} \mathrm{~s}^{-1}$ in the cored and $\sim 13 \mathrm{~km} \mathrm{~s}^{-1}$ in the cusped simulation from the average, which should be zero. We 
call these 'streams' fossil remnants of the formation process. With the advent of new techniques for astronomical observations, these streams should be detectable and can be used to test our formation scenario.
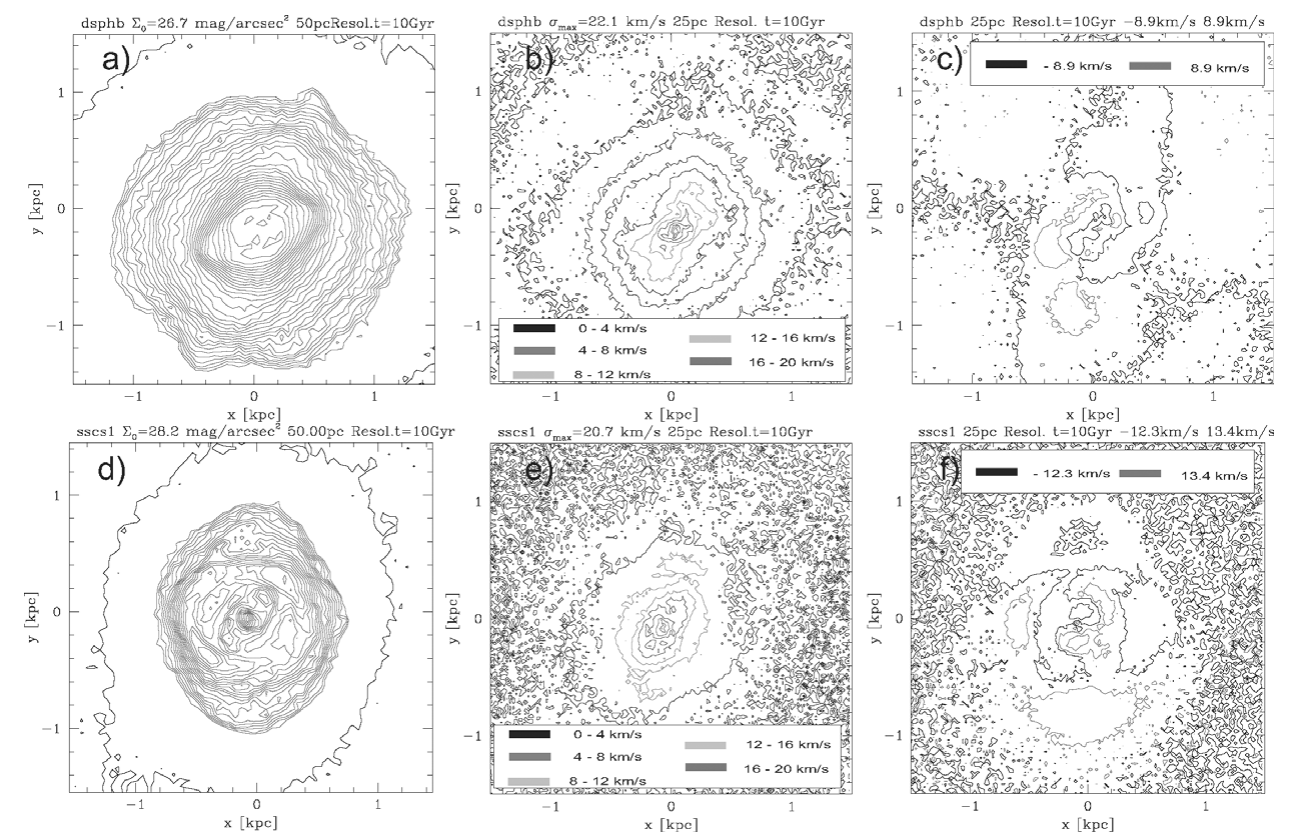

Figure 2. Projection of the dSph onto the $x y$ plane. In $(a)$ and $(d)$ the surface density is shown with $50 \mathrm{pc}$ resolution for the Plummer and NFW profiles, in (b) [(e)] the velocity dispersion with $25 \mathrm{pc}$ resolution for the Plummer [NFW] profile is shown. In $(c)$ and $(e)$ we show the mean velocities of the stars for the Plummer and NFW profiles.

The surface-brightness profile for the cored simulation after $10 \mathrm{Gyr}$ of evolution is shown in Figure 3 (left panel). We fit our model with King, Sérsic and Dehnen profiles. Our results are best described by the King profile. There, the core radius is $720 \mathrm{pc}$ and the central surface brightness is $26 \mathrm{mag} \operatorname{arcsec}^{-2}$. In the right panel of Figure 3 we show the line-of-sight velocity dispersion within a radius of $500 \mathrm{pc}$. The central velocity dispersion is $14.5 \mathrm{~km} \mathrm{~s}^{-1}$.

In Figure 4 we show some star clusters after 10 Gyr of evolution for the cusped simulation. The two star clusters shown represent the two main types of stellar streams we see. One from a radial orbit has spread its stars uniformly across a spherical region, while the other one, on a more circular orbit, has stars in a torus-like configuration. These toruslike 'streams' are responsible for an artificially raised velocity dispersion in projection. One also sees two surviving star clusters in the outskirts of the galaxy similar to the star clsuters in Sagittarius and Fornax.

\section{Conclusion and outlook}

We study numerically the formation of dSph galaxies from merging star clusters, considering two distinct types of profiles for the dark-matter halo. We compare the results of both simulations. We see that in the cusped simulation, stars are more centrally concentrated but have lower central velocity dispersions. In both simulations there are streams of stars (fossil remnants of dissolved star clusters) which survive more than 10 Gyr of evolution, raising the velocity dispersion artificially. With the advent of new techniques 

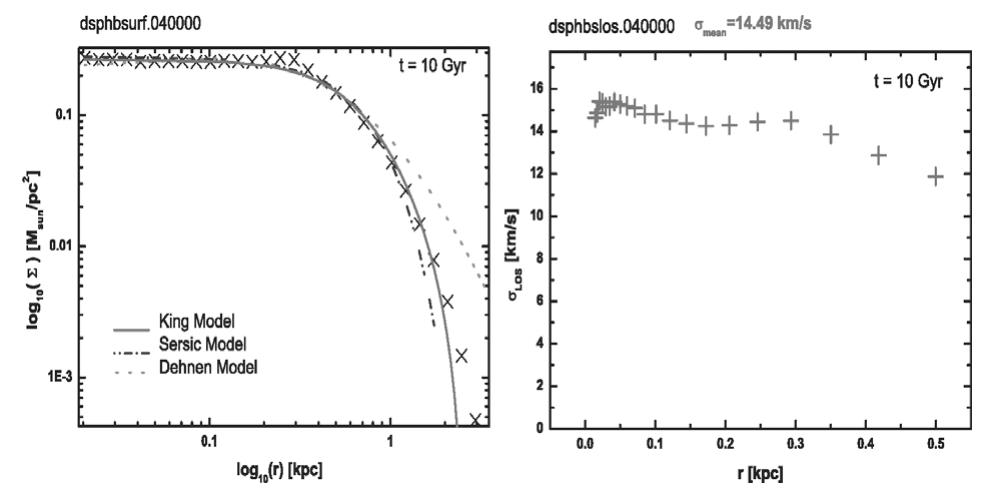

Figure 3. (a) Surface-density distribution fitted with different profiles. (b) Line-of-sight velocity-dispersion profile.

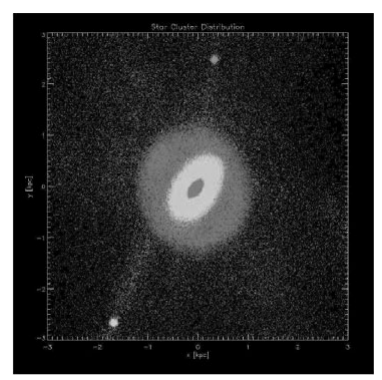

Figure 4. The red and yellow points represent two types of streams of stars at 10 Gyr in the central region of the dSph. We can also see two small star clusters orbiting the galaxy.

for astronomical observations, these streams should soon be detectable and can test our formation scenario. Our initial setup was chosen to produce a stellar component with a line-of-sight velocity dispersion of $10 \mathrm{~km} \mathrm{~s}^{-1}$, similar to the larger dSph galaxies of the Milky Way. But due to the stellar 'streams' we see an artificially raised dispersion in both models. These streams can only produce such an effect in a low-density environment like that of a dSph galaxy.

We will investigate this effect further and if we find that it is persistent substructure, we will explore the potential impact of undetected streams on estimates of the DM content of the Milky Way's dSph galaxies (based on measurements of the velocity dispersion).

\section{References}

Fellhauer, M., Kroupa, P., Baumgardt, H., Bien, R., Boily, C. M., Spurzem, R., \& Wassmer, N. 2000, NewA, 5, 305

Lada, C. J. \& Lada, E. A. 2003, ARA\& $A, 41,57$

Navarro, J. F., Frenk, C. S., \& White, S. D. M. 1997, ApJ, 490, 493

Plummer, H. C. 1911, MNRAS, 71, 460

Springel, V., et al. 2005, Nature, 435, 629 